\title{
Cerebral Microbleeds Are Associated with Loss of White Matter Integrity
}

\author{
(D).-Y. Liu, (D)Y.-J. Zhou, (D)F.-F. Zhai, (D)F. Han, (D).-X. Zhou, (D). Ni, (D) M. Yao, (D) S. Zhang, (D) Z. Jin, (D) L. Cui, and (D)Y.-C. Zhu
} ON $\equiv \nabla$

\begin{abstract}
BACKGROUND AND PURPOSE: Previous studies have shown that diffusion tensor imaging suggests a diffuse loss of white matter integrity in people with white matter hyperintensities or lacunes. The purpose of this study was to investigate whether the presence of cerebral microbleeds and their distribution are related to the integrity of white matter microstructures.
\end{abstract}

\begin{abstract}
MATERIALS AND METHODS: The study comprised 982 participants who underwent brain MR imaging to determine microbleed status. The cross-sectional relation between microbleeds and the microstructural integrity of the white matter was assessed by 2 statistical methods: a multilinear regression model based on the average DTI parameters of normal-appearing white matter and Tract-Based Spatial Statistics analysis, a tract-based voxelwise analysis. Fiber tractography was used to spatially describe the microstructural abnormalities along WM tracts containing a cerebral microbleed.
\end{abstract}

RESULTS: The presence of cerebral microbleeds was associated with lower mean fractional anisotropy and higher mean diffusivity, axial diffusivity, and radial diffusivity, and the association remained when cardiovascular risk factors and cerebral small-vessel disease markers were further adjusted. Tract-Based Spatial Statistics analysis indicated strictly lobar cerebral microbleeds associated with lower fractional anisotropy, higher mean diffusivity, and higher radial diffusivity in the internal capsule and corpus callosum after adjusting other cerebral small-vessel disease markers, while only a few voxels remained associated with deep cerebral microbleeds. Diffusion abnormalities gradients along WM tracts containing a cerebral microbleed were not found in fiber tractography analysis.

CONCLUSIONS: Cerebral microbleeds are associated with widely distributed changes in white matter, despite their focal appearance on SWI.

ABBREVIATIONS: $\mathrm{CMB}=$ cerebral microbleed; $\mathrm{CSVD}=$ cerebral small vessel disease; $\mathrm{MD}=$ mean diffusivity; $\mathrm{FA}=$ fractional anisotropy; NAWM $=$ normalappearing white matter; $\mathrm{WMH}=$ white matter hyperintensity; TBSS $=$ Tract-Based Spatial Statistics

A ccording to recent evidence, cerebral small vessel disease (CSVD) was found to have focal brain parenchymal lesions not only visible on brain MR imaging but also related to a wider range of white matter microstructural injury. ${ }^{1-3}$ These studies mainly focused on ischemic lesions related to CSVD, such as lacunes or white matter hyperintensities.

Cerebral microbleeds (CMBs), characterized as focal hemosiderin deposits without remarkable brain tissue loss in

Received February 8, 2020; accepted after revision May 1.

From the Departments of Neurology (J.-Y.L., Y.-J.Z., F.-F.Z., F.H., L.-X.Z., J.N., M.Y., L.C., Y.-C.Z.), Radiology (Z.J.), and Cardiology (S.Z.), Peking Union Medical College Hospital, Peking Union Medical College and Chinese Academy of Medical Science, Beijing, China.

J.-Y. Liu and Y.-J. Zhou contributed equally to the article.

This study was funded by National Key Research and Development Program of China (grant No. 2017YFE0100500), National Natural Science Foundation of China (grant No. 81671173), Strategic Priority Research Program (pilot study) "Biological Basis of Aging and Therapeutic Strategies" of the Chinese Academy of Sciences (grant No. XDPB10), and the CAMS Innovation Fund for Medical Sciences (No. 2017-I2M-3-008)

pathologic studies, are the only hemorrhagic marker of CSVD and apparently different from white matter hyperintensities (WMHs) and lacunes. Theoretically, CMBs might also be associated with remote or extensive lesions of the brain because CMBs appear as markers of hypertension and cerebral amyloid antipathy, which can lead to the dysfunction of the blood-brain barrier, erythrocyte extravasation, and diffuse influence on brain tissue. ${ }^{4-6}$ Akoudad et $\mathrm{al}^{7}$ found, in the Rotterdam Scan Study, that the presence of CMBs was associated with lower mean fractional anisotropy (FA) and higher mean diffusivity (MD) in DTI,

Please address correspondence to Yi-Cheng Zhu, Department of Neurology, Peking Union Medical College Hospital, Peking Union Medical College and Chinese Academy of Medical Science, No.1 Shuaifuyuan, Wangfujing, Beijing, 100730, China; e-mail: zhuych910@163.com

- Indicates open access to non-subscribers at www.ajnr.org

三 Indicates article with supplemental on-line appendix and table.

Indicates article with supplemental on-line photo.

http://dx.doi.org/10.3174/ajnr.A6622 


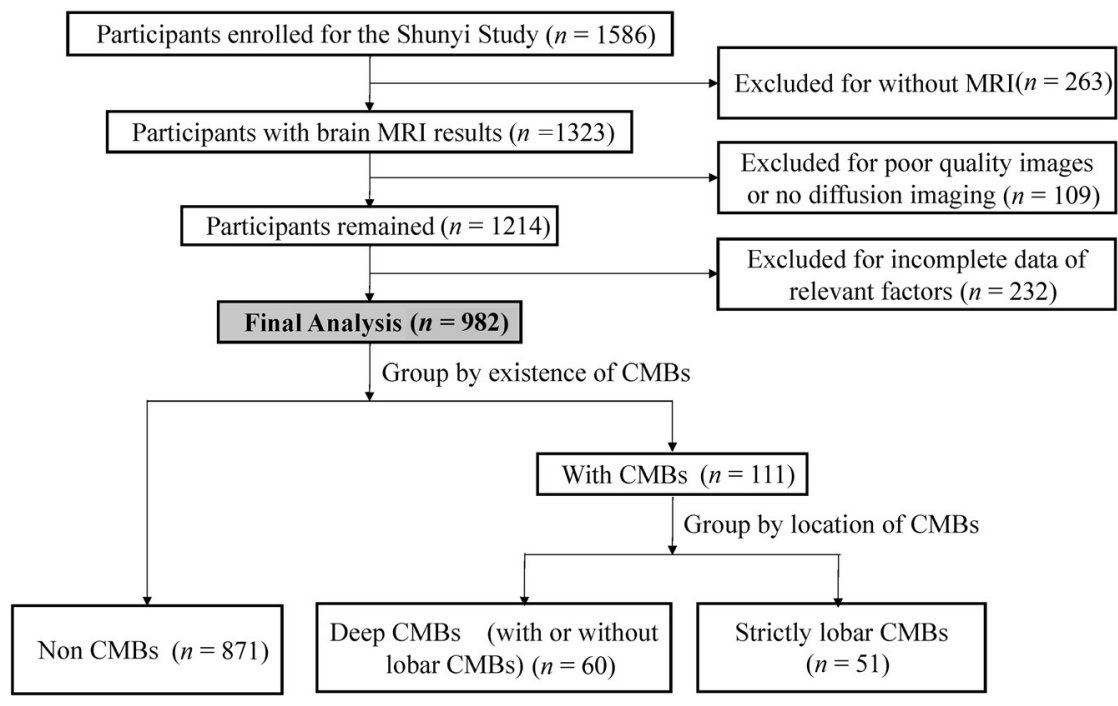

FIG 1. Flow diagram of the study population.

supporting the above-mentioned hypothesis. However, answers to serious questions remained unclear. First, few studies further investigated the dimensional distribution of microstructural integrity changes that related to different distribution patterns of CMBs, which imply different pathologic types of CSVD. Moreover, a previous study demonstrated that diffusion abnormalities gradients surround lacunes, suggesting a perilesional effect. ${ }^{8}$ Whether CMBs can also lead to a similar perilesional effect remained unclear.

To answer the questions mentioned above, we investigated by DTI whether the presence and distribution of CMBs were associated with the destruction of white matter integrity on the basis of a community-based sample, and we further used fiber tractography to spatially describe the microstructural abnormalities along white matter tracts containing a CMB.

\section{MATERIALS AND METHODS \\ Participants}

This study is embedded in the Shunyi study, an ongoing prospective population-based cohort study that aims to investigate the risk factors and consequences of brain changes in communitydwelling adults in a Chinese population. All inhabitants 35 years of age or older who independently lived in 5 villages of Shunyi, a suburb of Beijing, were invited to participate in this study. A total of 1586 participants agreed to join between June 2013 and April 2017. Standard clinical examinations were performed for each participant at baseline, including structured questionnaires, physical examinations, and blood tests. All participants were advised to complete a brain MR imaging examination, except those who refused or had contradictions for MR imaging, a total of 263. Thus, 1323 participants underwent brain MR imaging. The study was approved by the ethics committee at Peking Union Medical College Hospital (reference No. B-160). Written informed consent was obtained from all participants.

Among the 1323 participants who underwent brain MR imaging, those with poor-quality images and no diffusion imaging $(n=109)$ and those with missing data of vascular risk factors and other baseline characteristics ( $n=$ 232) were excluded, leaving 982 subjects used for data analysis (Fig 1). The baseline characteristics of the participants included and not included in the current study were balanced.

\section{Vascular Risk Factors}

Cardiovascular risk factors were collected using structured questionnaires, physical examinations, and blood tests. Definitions of cardiovascular risk factors are described in the On-line Appendix.

\section{MR Imaging}

A 3T Magnetom Skyra scanner (Siemens) was used for the brain MR imaging examination. 3D-T1-weighted, T2-weighted, FLAIR, and SWI were performed as described in detail previously. ${ }^{9}$

All imaging markers were rated and defined on the basis of the Standards for Reporting Vascular Changes on Neuroimaging. ${ }^{10}$ Lacunes were defined as areas with a diameter $>3$ $\mathrm{mm}$ and $<15 \mathrm{~mm}$ with low signal intensity on T1 and FLAIR and high signal intensity on T2, located at the basal ganglia or white matter. CMBs were defined as small, round, or ovoid hypointense lesions found on the SWI sequence and categorized into 2 groups according to their location: strictly lobar CMBs and deep CMBs. Strictly lobar CMBs were defined as CMBs located at the cortical gray and subcortical or periventricular white matter. Deep CMBs were defined as CMBs located at other places (deep gray matter including the basal ganglia and thalamus; white matter of the corpus callosum, internal, external, and extreme capsules; brain stem and cerebellum), with or without lobar CMBs. White matter hyperintensities were segmented by the lesiongrowth algorithm ${ }^{11}$ as implemented in the LST toolbox (https:// www.applied-statistics.de/lst.html) for Statistical Parametric Mapping 12 (http://www.fil.ion.ucl.ac.uk/spm/). The gray matter, white matter, and CSF were segmented using Statistical Parametric Mapping 12. Total brain volume was computed as the sum of gray matter and white matter volumes.

Lacunes and CMBs were rated by 2 well-trained neurologists who were blinded to clinical data, independently (F.H. for lacunes and Q.W. for CMBs). Intrarater agreement was assessed in a random sample of 50 individuals with an interval of $>1$ month between the first and second readings. $\kappa$ values for the intrarater agreements were 0.95 for lacunes and 0.90 for CMBs.

\section{Diffusion Imaging Analysis}

Details on the diffusion imaging processing are listed in the On-line Appendix. Diffusion tensor images were processed using PANDA, a pipeline toolbox for diffusion MR imaging analysis (https://www.nitrc.org/projects/panda/). ${ }^{12} \mathrm{FA}, \mathrm{MD}$, axial diffusivity, and radial diffusivity maps in native space 


\begin{tabular}{|c|c|c|c|c|c|}
\hline Variables & Normal & Deep CMBs & Lobar CMBs & $P^{\mathrm{b}}$ & $P^{c}$ \\
\hline \multicolumn{6}{|l|}{ Demographics } \\
\hline Age (yr) & $55.3(9.1)$ & $62.6(7.5)$ & $60.8(9.0)$ & $<.001$ & .314 \\
\hline Men & $296(34 \%)$ & $36(60 \%)$ & $20(39 \%)$ & $<.001$ & .048 \\
\hline \multicolumn{6}{|l|}{ Cardiovascular risk factors } \\
\hline Hypertension & 427 (49\%) & $48(80 \%)$ & 27 (53\%) & $<.001$ & $.002^{\mathrm{b}}$ \\
\hline Diabetes mellites & 148 (17\%) & $14(23 \%)$ & $12(24 \%)$ & .212 & .981 \\
\hline Hyperlipidemia & 427 (49\%) & $29(48 \%)$ & $27(53 \%)$ & .869 & .628 \\
\hline Smoking & $231(26.5 \%)$ & $25(41.7 \%)$ & $15(29.4 \%)$ & .038 & .180 \\
\hline \multicolumn{6}{|l|}{ Neuroimaging characteristics } \\
\hline Presence of lacunes & $122(14 \%)$ & 35 (58\%) & 15 (29\%) & $<.001$ & $.002^{\mathrm{d}}$ \\
\hline WMH volume $(\mathrm{mL})$ & $0.82(0.25-2.62)$ & $5.82(2.09-12.81)$ & $3.56(0.99-7.11)$ & $<.001$ & .086 \\
\hline Total brain volume $(\mathrm{mL})$ & $1402.7(123.2)$ & $1421.0(132.6)$ & $1424.2(136.3)$ & .285 & .893 \\
\hline CMB numbers & & & & - & $.003^{\mathrm{d}}$ \\
\hline 1 & - & $29(48.3 \%)$ & $36(70.5 \%)$ & & \\
\hline $2 \sim 3$ & - & $17(28.3 \%)$ & $13(25.5 \%)$ & & \\
\hline$>4$ & - & $14(23.3 \%)$ & $2(4.0 \%)$ & & \\
\hline \multicolumn{6}{|l|}{ DTI parameters } \\
\hline Mean global FA, NAWM & $0.37(0.02)$ & $0.35(0.03)$ & $0.35(0.02)$ & $<.001$ & .143 \\
\hline Mean global MD, $\times 10^{-3} \mathrm{~mm}^{2} / \mathrm{s}$, NAWM & $0.84(0.04)$ & $0.89(0.06)$ & $0.87(0.05)$ & $<.001$ & .031 \\
\hline Axial diffusivity, $\times 10^{-3} \mathrm{~mm}^{2} / \mathrm{s}$, NAWM & $1.18(0.04)$ & $1.22(0.05)$ & $1.20(0.05)$ & $<.001$ & .023 \\
\hline Radial diffusivity, $\times 10^{-3} \mathrm{~mm}^{2} / \mathrm{s}$, NAWM & $0.67(0.05)$ & $0.73(0.07)$ & $0.71(0.06)$ & $<.001$ & .042 \\
\hline
\end{tabular}

Note:--indicates no CMBs.

${ }^{a}$ Data represent mean (SD), median (interquartile range), or frequency (percentage).

b Significance test among 3 groups, using the ANOVA, $\chi^{2}$ test, or the Kruskal-Wallis test.

' Post hoc analysis between the deep CMB and lobar CMB groups.

${ }^{\mathrm{d}}$ The difference was significant between deep CMBs and lobar CMBs groups (Bonferroni-corrected, $P<.017$ ).

and standard Montreal Neurological Institute space were generated for each individual. Furthermore, diffusion parameters in normal-appearing white matter (NAWM) were also calculated using NAWM masks created by excluding the individual WMH segmentation mask from a mean white matter mask.

Then, we performed Tract-Based Spatial Statistics (TBSS; http://fsl.fmrib.ox.ac.uk/fsl/fslwiki/TBSS) to assess the association between CMBs and white matter integrity. ${ }^{13}$ TBSS analysis was performed for 2 groups according to the location of cerebral microbleeds (none versus strictly lobar CMBs; none versus deep CMBs). TBSS was performed using a permutation-based statistical interference tool for nonparametric analyses as part of the FMRIB Software Library toolbox (https://fsl.fmrib.ox.ac. $\mathrm{uk} / \mathrm{fs}$ //fslwiki/Randomise). The number of permutation tests was set at 5000, and significant thresholds were determined using a threshold-free cluster enhancement with $P$ value $<.05$ to correct for multiple testing.

Along-tract statistics (https://github.com/johncolby/along-tractstats) were used to spatially describe the microstructural abnormalities along WM tracts containing a CMB. Those who had $>1 \mathrm{CMB}$ and had CMBs near lacunes or WMHs were excluded, leaving 20 participants with only $1 \mathrm{CMB}$ in the supratentorial white matter. Whole-brain deterministic tractography was performed according to the FACT algorithm, ${ }^{14}$ as implemented in Diffusion Toolkit v0.6 (http://www.trackvis.org/dtk). Tracking constraints included a minimum FA threshold of 0.2 and a maximum fiber-turning angle of $45^{\circ}$. For each microbleed, the fiber containing the microbleed and the corresponding fiber at the contralateral hemisphere were extracted manually. Fiber tract diffusion properties (FA and MD) were investigated along the tract at 5-mm intervals. ${ }^{15}$ Comparisons were made between the affected and control tracts for each segment using paired $t$ tests (On-line Figure).

\section{Statistical Analyses}

The baseline characteristics were presented as mean $( \pm S D)$, median (interquartile range), or frequency (percentage). Baseline characteristics were compared among groups using ANOVA, a nonparametric test, or a $\chi^{2}$ test. Cerebral microbleed status was investigated dichotomously (none versus $\geq 1$ $\mathrm{CMB}$ ), and by location (none versus strictly lobar CMBs; none versus deep CMBs). Multiple linear regression was used to evaluate the association between microbleed status and the integrity of brain white matter in 4 models. WMH volume was natural log-transformed because of its skewed distribution. Subject-specific mean FA, mean MD, and axial and radial diffusivities were standardized into $z$ scores. In model 1 , we adjusted for age and sex. In model 2, we additionally adjusted for cardiovascular risk factors (hypertension, diabetes mellitus, hyperlipidemia, smoking). In model 3, we adjusted for age, sex, and other imaging markers of CSVD, including WMH volume and the presence of lacunes. In model 4, we adjusted for both cardiovascular risk factors and other imaging markers of CSVD. All analyses were performed using the statistical software package SPSS 23.0 (IBM) and an $\alpha$ value of .05.

\section{RESULTS}

Table 1 provides the characteristics of the study population. In 871 participants without CMBs, the mean age was 55.3 years, and 296 participants were men. Fifty-one participants had strictly lobar microbleeds, and 60 had deep microbleeds. The portion of men was relatively higher $(60 \%)$ in participants with deep microbleeds. Compared with the strictly lobar microbleeds group, the proportion of patients with deep microbleeds with hypertension and lacunes was higher. 
Table 2: Microbleeds (yes versus no) and white matter microstructure integrity ${ }^{\mathrm{a}}$

\begin{tabular}{|c|c|c|c|c|c|c|c|c|}
\hline & \multicolumn{2}{|l|}{ FA } & \multicolumn{2}{|l|}{ MD } & \multicolumn{2}{|c|}{ Axial Diffusivity } & \multicolumn{2}{|c|}{ Radial Diffusivity } \\
\hline & $\beta$ & $P$ & $\beta$ & $P$ & $\beta$ & $P$ & $\beta$ & $P$ \\
\hline Model 1 & $-0.450(-0.616$ to -0.285$)$ & $<.001$ & $0.433(0.282-0.584)$ & $<.001$ & $0.378(0.221-0.535)$ & $<.001$ & $0.446(0.295-0.597)$ & $<.001$ \\
\hline Model 2 & $-0.433(-0.597$ to -0.269$)$ & $<.001$ & $0.420(0.271-0.570)$ & $<.001$ & $0.368(0.212-0.524)$ & $<.001$ & $0.432(0.282-0.581)$ & $<.001$ \\
\hline Model 3 & $-0.238(-0.394$ to -0.083$)$ & .003 & $0.204(0.068-0.340)$ & .003 & $0.165(0.019-0.310)$ & .026 & $0.217(0.081-0.352)$ & .002 \\
\hline Model 4 & $-0.241(-0.396$ to -0.086$)$ & .002 & $0.206(0.070-0.342)$ & .003 & $0.167(0.021-0.312)$ & .025 & $0.218(0.083-0.354)$ & .002 \\
\hline
\end{tabular}

Note: $-\beta$ indicates regression coefficient.

${ }^{a}$ Values represent differences in $z$ scores of mean FA, MD, axial diffusivity, and radial diffusivity of NAWM for the presence of any microbleeds compared with no microbleeds. Model 1: adjusted for age and sex; model 2: same as model 1, additionally adjusted for hypertension, hyperlipidemia, smoking, diabetes; model 3: adjusted for age, sex, lacunes, and white matter hyperintensity volume (log-transformed); model 4: adjusted for sex, cardiovascular risk factors as in model 2, and CSVD imaging markers as in model 3.

Table 3: Microbleeds by location and white matter microstructure integrity ${ }^{\mathrm{a}}$

\begin{tabular}{|c|c|c|c|c|c|c|c|c|}
\hline & \multicolumn{2}{|l|}{ FA } & \multicolumn{2}{|l|}{ MD } & \multicolumn{2}{|l|}{ Axial Diffusivity } & \multicolumn{2}{|c|}{ Radial Diffusivity } \\
\hline & $\beta$ & $P$ & $\beta$ & $P$ & $\beta$ & $P$ & $\beta$ & $P$ \\
\hline \multicolumn{9}{|l|}{$\begin{array}{c}\text { None vs } \\
\text { lobar }\end{array}$} \\
\hline Model 1 & $-0.396(-0.622$ to -0.169$)$ & .001 & $0.339(0.135-0.543)$ & .001 & $0.270(0.055-0.484)$ & .014 & $0.363(0.159-0.566)$ & $<.001$ \\
\hline Model 2 & $-0.396(-0.619$ to -0.173$)$ & .001 & $0.340(0.138-0.541)$ & .001 & $0.270(0.058-0.485)$ & .013 & $0.362(0.162-0.563)$ & $<.001$ \\
\hline Model 3 & $-0.258(-0.467$ to -0.048$)$ & .016 & $0.191(0.010-0.372)$ & .038 & $0.131(-0.065-0.327)$ & .191 & $0.215(0.034-0.395)$ & .020 \\
\hline Model 4 & $-0.264(-0.472$ to -0.056$)$ & .013 & $0.195(0.014-0.376)$ & .034 & $0.134(-0.062-0.330)$ & .180 & $0.218(0.039-0.398)$ & .017 \\
\hline \multicolumn{9}{|l|}{$\begin{array}{l}\text { None vs } \\
\text { deep }\end{array}$} \\
\hline Model 1 & $-0.509(-0.726$ to -0.293$)$ & $<.001$ & $0.530(0.335-0.725)$ & $<.001$ & $0.486(0.283-0.689)$ & $<.001$ & $0.534(0.339-0.729)$ & $<.001$ \\
\hline Model 2 & $-0.481(-0.697$ to -0.266$)$ & .001 & $0.512(0.317-0.706)$ & $<.001$ & $0.472(0.268-0.675)$ & $<.001$ & $0.514(0.319-0.708)$ & $<.001$ \\
\hline Model 3 & $-0.244(-0.450$ to -0.038$)$ & .020 & $0.243(0.065-0.421)$ & .008 & $0.218(0.028-0.408)$ & .025 & $0.246(0.068-0.424)$ & .007 \\
\hline Model 4 & $-0.242(-0.448$ to -0.036$)$ & .021 & $0.245(0.071-0.424)$ & .007 & $0.220(0.029-0.411)^{\prime}$ & .024 & $0.248(0.069-0.426)$ & .007 \\
\hline
\end{tabular}

Note: $-\beta$ indicates regression coefficient.

${ }^{a}$ Values represent differences in z scores of mean FA, MD, axial diffusivity and radial diffusivity of NAWM for the presence of any microbleeds by their location compared with no microbleeds. Model 1: adjusted for age and sex; model 2: same as model 1, additionally adjusted for hypertension, hyperlipidemia, smoking, diabetes; model 3: adjusted for age, sex, lacunes, white matter hyperintensity volume (log-transformed); model 4: adjusted for sex, cardiovascular risk factors as in model 2, and CSVD imaging markers as in model 3.

\section{Association between CMBs and White Matter Integrity}

The association between the presence of CMBs and DTI measurements is shown in Table 2. When adjusted for age and sex, participants with $\mathrm{CMBs}$ had reduced FA and higher MD, axial diffusivity, and radial diffusivity (all $P<.001$, model 1 ) The association remained when cardiovascular risk factors and CSVD markers were further adjusted (models 2, 3, and 4).

Table 3 reveals the association between DTI measurements and different $\mathrm{CMB}$ locations. Significantly lower mean FA and higher mean $\mathrm{MD}$, axial diffusivity, and radial diffusivity were observed in models 1 and 2 in participants with deep CMBs and those with strictly lobar CMBs. With additional adjustment for CSVD markers, the association remained in participants with deep CMBs (model 4). In participants with lobar CMBs, such associations remained after adjustment for markers of CSVD, except that no significant association was identified between lobar CMBs and axial diffusivity (model 4 : $\beta=0.134$; 95\% CI, $-0.062 \sim 0.330 ; P=.180)$.

\section{Tract-Based Spatial Statistics Analysis}

As shown in Fig $2 A$, the DTI measurements at almost all voxels on the skeleton were significantly different between participants with deep CMBs and healthy participants. Similar differences of the DTI measurements between participants with strictly lobar CMBs and healthy participants were also found, as shown in Fig $3 A$, except that in only a few voxels was the higher axial diffusivity associated with lobar CMBs. With additional control of CSVD imaging markers, the relations of deep CMBs and DTI parameters disappeared, except that participants with deep CMBs had a higher MD and higher radial diffusivity in some voxels located at the anterior limb of the internal capsule and genu of the corpus callosum (Fig 2B). In the strictly lobar CMB group, lower FA, higher $\mathrm{MD}$, and higher radial diffusivity were found at the genu of the corpus callosum and the anterior limb of the internal capsule compared with those in healthy groups, but no significant differences of axial diffusivity were identified between them

(Fig 3B).

\section{Along-Tract Statistics Analysis}

Detailed information of the selected subjects is given in the Online Table. As shown in Fig 4, there was no detectable change in FA $(\mathrm{CMB} /$ control $=0.987, P=.429)$ and $\mathrm{MD}(\mathrm{CMB} /$ control $=$ $1.021, P=.225)$ at the $\mathrm{CMB}$ location. No detectable change was found as the distance increased from the CMB location.

\section{DISCUSSION}

In this prospective population-based cohort study, we found that the presence of CMBs, whether deep or lobar, was associated with lower mean FA and higher mean MD. The voxelwise TBSS analysis showed that after adjusting for other CSVD MR imaging markers, multiple regions of the white matter skeleton (mainly in the anterior limb of the internal capsule and the genu of the corpus callosum) were associated with strictly lobar cerebral microbleeds, while only a few voxels remained to be associated with 


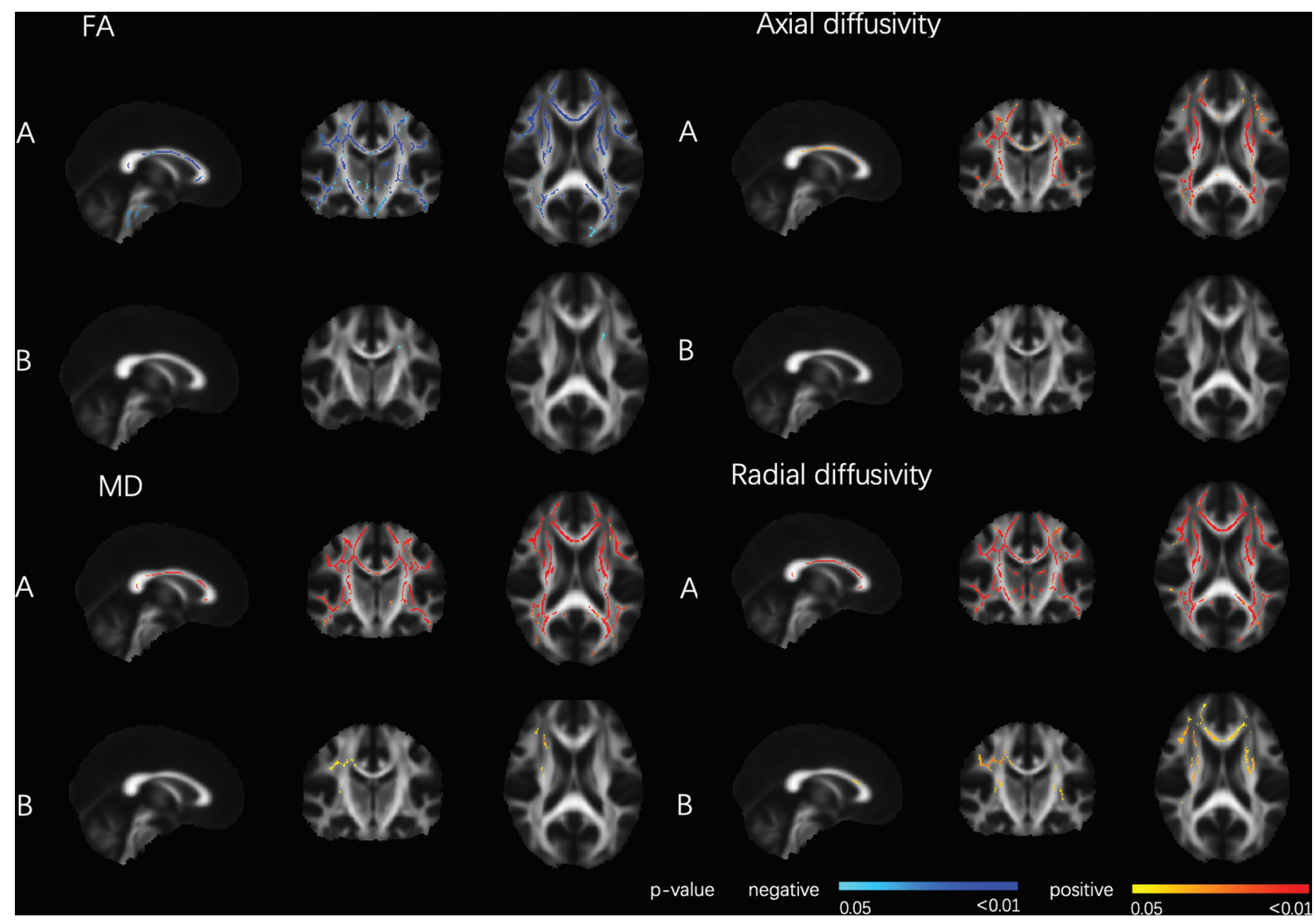

FIG 2. DTI parameters related to deep CMBs. Tract-Based Spatial Statistics analysis of FA and the CMB location (deep CMBs with or without lobar CMBs). Deep CMBs were associated with decreased FA and increased MD, axial diffusivity, and radial diffusivity, adjusted for age, sex, hypertension, hyperlipidemia, smoking, and diabetes $(A)$, or age, sex, hypertension, hyperlipidemia, smoking, diabetes, white matter hyperintensity volume, and lacunes $(B)$, thresholded at $P<.05$, and corrected for multiple comparisons. The red colormap indicates a positive relationship, and the blue colormap indicates a negative relationship. The statistical maps are superimposed onto the spatially normalized (Montreal Neurological Institute stereotactic space) and averaged ( $n=931)$ FA map. Threshold-free cluster enhancement corrected $P<.05$.

deep CMBs. Diffusion abnormalities gradients along WM tracts containing a CMB were not found in fiber tractography analysis.

We found that the presence of CMBs was associated with lower mean FA and higher mean MD after adjusting for crucial vascular risk factors and other CSVD MR imaging markers. This result was consistent with a previous study by Akoudad et al, ${ }^{7}$ in 2013, indicating that the presence of CMBs was associated with the loss of white matter structural integrity. We found that both radial diffusivity and axial diffusivity were higher in the $\mathrm{CMB}$ group, a finding also consistent with those of Akoudad et al. At present, it is thought that the increase in radial diffusivity may reflect the increase of extra-axonal fluid caused by demyelination or destruction of myelin integrity, while the decrease of axial diffusivity may mean the loss of axons and the destruction of fiber consistency. ${ }^{3,16}$ Does this mean that CMBs are mainly associated with myelin sheath loss in the white matter? Because the relation between the pattern of changes in DTI metrics and pathologic changes is mostly observed in animal models, the exact pathologic processes leading to changes in the diffusion tensors in the human brain still need to be further explored.

The TBSS result in our study that white matter microstructure destruction was found to be associated with strictly lobar microbleeds after adjusting CSVD markers, while only few voxels remained to be associated with deep microbleeds after adjusting CSVD markers, suggested that deep CMBs and lobar CMBs are related to the damage of white matter microstructures through different mechanisms. It is believed that deep microbleeds are related to hypertension arteriopathy, while lobar microbleeds result primarily from cerebral amyloid angiopathy. ${ }^{5}$ The previous study by Akoudad et $\mathrm{al}^{7}$ claimed that allele Apolipoprotein E4 carriers with strictly lobar microbleeds had poorer white matter integrity than noncarriers with strictly lobar CMBs, and effect modification by Apolipoprotein E4 carriership was not present for deep microbleeds, suggesting that amyloid angiography itself might play an important role in the relationship between strictly lobar CMBs and diffuse white matter damage. In addition, a recent study that investigated the histopathology of diffusion imaging abnormalities in 9 patients with cerebral amyloid angiopathy found that axonal and myelin loss are major components underlying cerebral amyloid angiopathy-related alterations in DTI properties observed in living patients. ${ }^{17}$ The discrepancy in the relation between the deep CMBs and DTI parameters displayed by a multilinear regression model based on the average DTI parameters and the tract-based voxelwise TBSS analysis may 


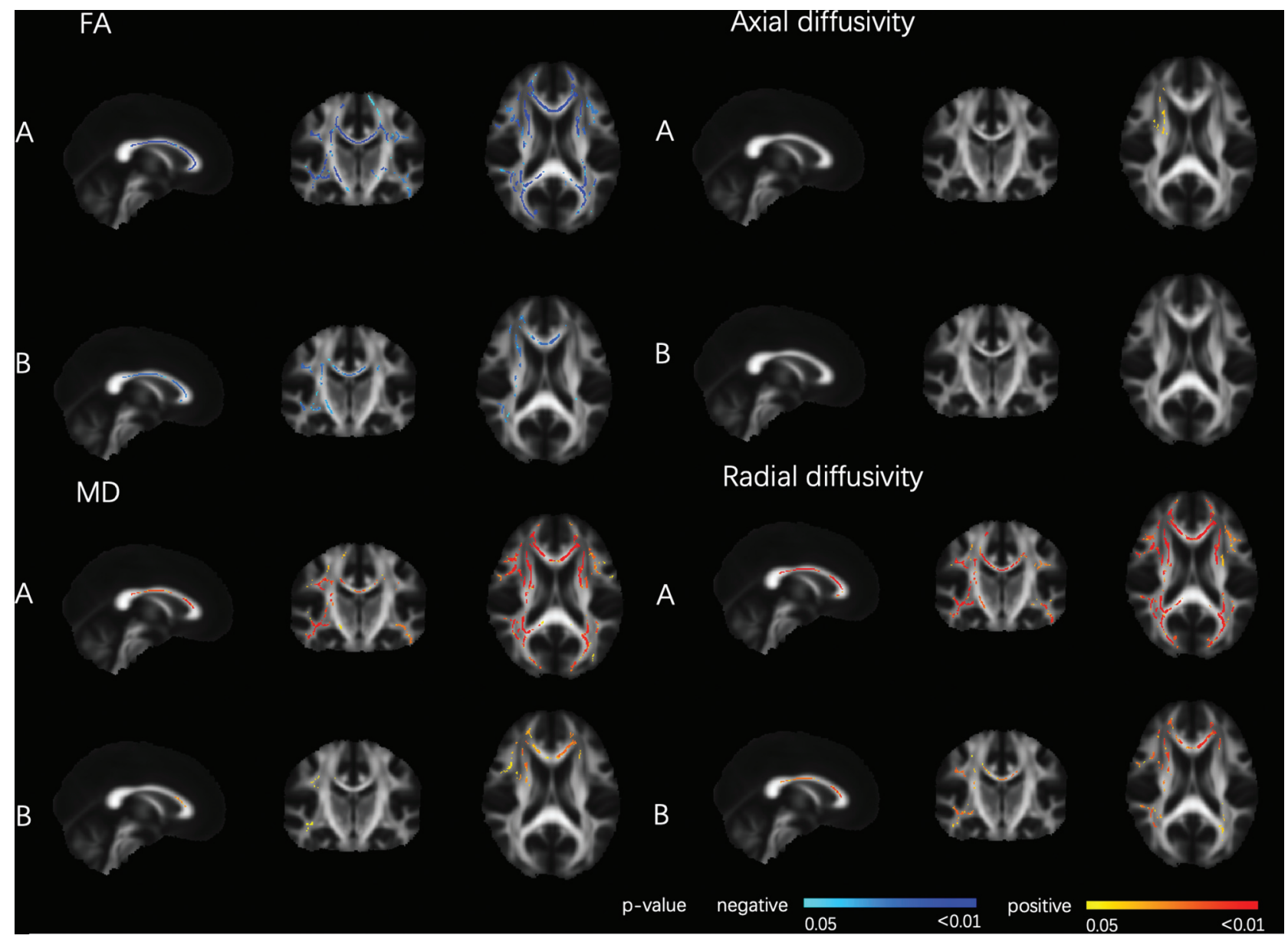

FIG 3. DTI parameters related to strictly lobar CMB. Tract-Based Spatial Statistics analysis of FA and CMB location (strictly lobar). Lobar CMBs were associated with decreased FA and increased MD, axial diffusivity, and radial diffusivity, adjusted for age, sex, hypertension, hyperlipidemia, smoking, and diabetes $(A)$, or age, sex, hypertension, hyperlipidemia, smoking, diabetes, white matter hyperintensity volume and lacunes (B), thresholded at $P<.05$ and corrected for multiple comparisons. The red colormap indicates a positive relationship, and the blue colormap indicates negative relationship. The statistical maps are superimposed onto the spatially normalized (Montreal Neurological Institute stereotactic space) and averaged ( $n=922)$ FA map. Threshold-free cluster enhancement corrected $P<.05$.

also be due to the influence of the extreme value on the average DTI parameters used for multiple linear regression.

Several theories can be proposed to explain the relation between lobar CMBs and changes of DTI parameters in the internal capsule and corpus callosum: White matter fibers in these regions are relatively dense, which may lead to cumulative effects of white matter damage in these parts. Alternatively, because the fibers in the genu of the corpus callosum and the anterior limb of the internal capsule are mainly connected with the frontal lobe, lobar microbleeds may be related to the damage of white matter fibers originating from the frontal lobe. Follow-up studies can provide a more detailed anatomic division of CMBs and assess the integrity of major white matter fiber tracts using diffusion tensor tractography to analyze the relationship between CMB topography and white matter damage. ${ }^{18}$

There are several hypotheses about the mechanism underlying the correlation between CMBs and white matter integrity loss. First, it is believed that microbleeds are mainly caused by impaired $\mathrm{BBB}$, which can also lead to other diffuse pathologic changes, including white matter rarefication. ${ }^{19}$ Second, vascular risk factors lead to pathologic vascular changes. CMBs and white matter integrity damage may occur together as downstream products. $^{9,20-22}$ There was also the hypothesis that CMBs might directly damage the adjacent white matter. However, we did not find a gradient of diffusion abnormalities surrounding the CMBs, indicating that perilesional damage might not be the cause of CMB-related white matter integrity loss. This possibility suggests that the white matter damage associated with CMBs is not confined to the site of microbleeds, but is diffuse.

Our results support the hypothesis that microbleeds reflect a more extensive impact despite their focal appearance on SWI. Therefore, the presence of microbleeds may be an important marker of CSVD risk stratification in clinical practice. The association between CMBs and diffuse white matter damage remained after adjusting for WMHs and lacunes, which may reflect different underlying pathologic mechanisms between CMBs and other CSVD markers. The differential description of their pathologic mechanisms associated with the loss of white matter integrity may have an impact on the treatment strategy.

A large sample size and population-based settings make this study more generalizable, the main advantage of our research. In addition, a comprehensive, unified protocol and high-quality 

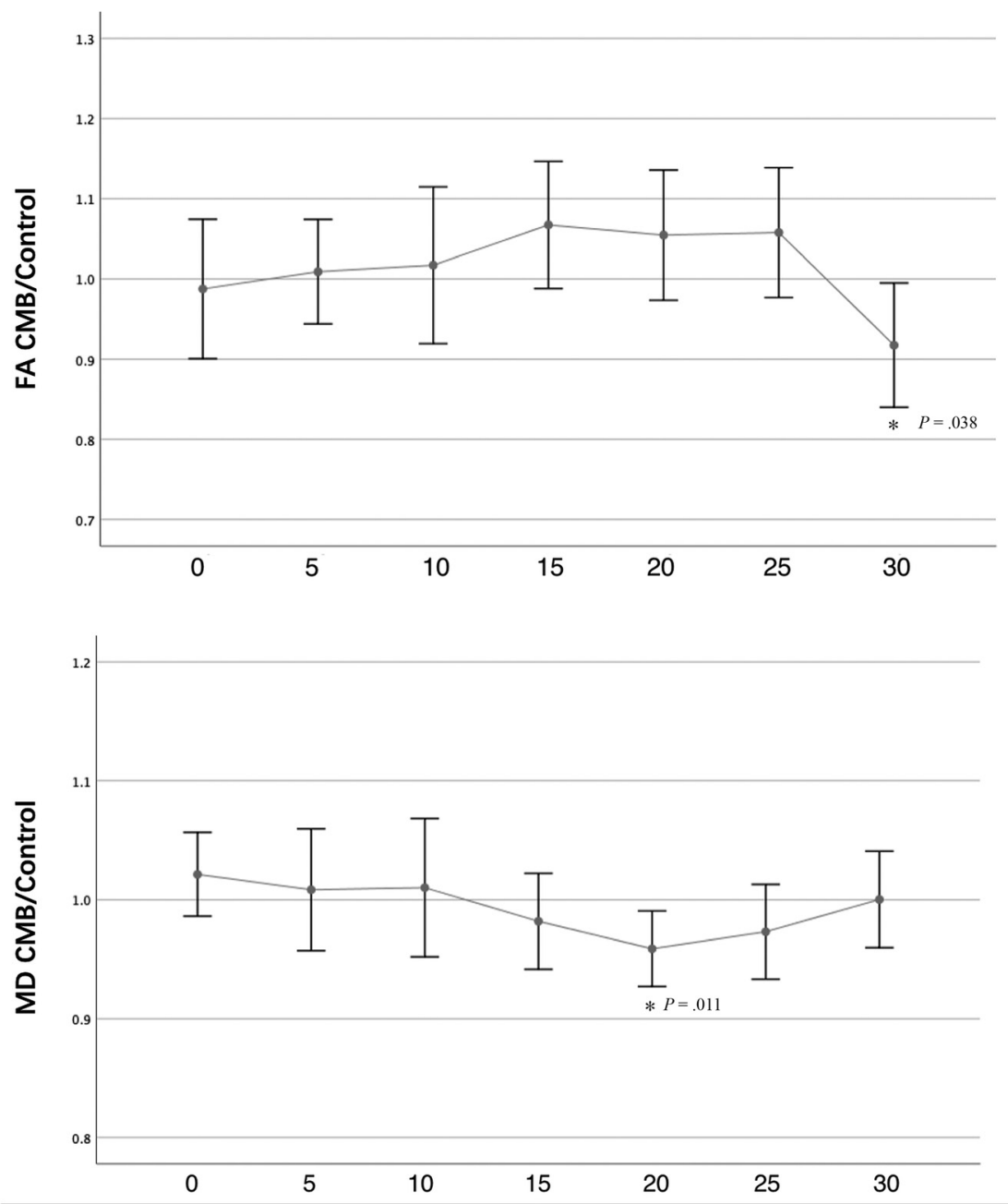

FIG 4. FA and MD of the lesioned tract expressed as a ratio relative to the control tract. The $x$-axis shows the distance in millimeters from the microbleed location. Data show the mean and its $95 \% \mathrm{CI}$. The asterisk indicates that the difference was significant between the $\mathrm{CMB}$ tract and control tract $(P<.05)$.

clinical MR imaging data have increased the reliability of this study. To avoid the influence of WMH on the results, we calculated the average DTI parameters of NAWM; this calculation was not performed in most previous studies. However, some limitations of our study need to be mentioned. First, our results are based on cross-sectional data, which prevent us from making a causal inference. Because our study is based on a cohort study, long-term follow-up can be conducted to observe the effects of $\mathrm{CMB}$ progression on white matter changes. Second, this article aims to reveal the relationship between cerebral microbleed location and white matter microstructure. However, the severity of cerebral microbleeds may also influence the integrity of white matter. Because the sample size of the participants with $>4$ CMBs was limited, further research is needed to investigate the association between the severity of CMBs and white matter microstructure.

\section{CONCLUSIONS}

Our results support the hypothesis that CMBs are associated with widely distributed changes in white matter despite their focal appearance on SWI. This finding further enriches our understanding that the lesions of CSVD are more diffuse than what can be seen on conventional MR imaging.

Disclosures: Yi-Cheng Zhu-RELATED: Grant: National Key Research and Development Program of China (No. 2017YFE0100500), National Natural Science Foundation of China (No. 81671173), Strategic Priority Research Program (pilot study) "Biological Basis of aging and Therapeutic Strategies" of the Chinese Academy of Sciences (No. XDPB10), the CAMS Innovation Fund for Medical Sciences (No. 2017I2M-3-008).* *Money paid to the institution.

\section{REFERENCES}

1. O'Sullivan M, Morris RG, Huckstep B, et al. Diffusion tensor MRI correlates with executive dysfunction in patients with ischaemic leukoaraiosis. J Neurol Neurosurg Psychiatry 2004;75:441-47 Medline

2. Auriel E, Edlow BL, Reijmer YD, et al. Microinfarct disruption of white matter structure: a longitudinal diffusion tensor analysis. Neurology 2014;83:182-88 CrossRef Medline

3. Sen PN, Basser PJ. A model for diffusion in white matter in the brain. Biophys J 2005;89:2927-38 CrossRef Medline

4. Fisher M. Cerebral microbleeds: where are we now? Neurology 2014;83:1304-05 CrossRef Medline

5. Greenberg SM, Vernooij MW, Cordonnier C, et al; Microbleed Study Group. Cerebral microbleeds: a guide to detection and interpretation. Lancet Neurol 2009;8:165-74 CrossRef Medline 
6. Renard D. Cerebral microbleeds: a magnetic resonance imaging review of common and less common causes. Eur J Neurol 2018; 25:441-50 CrossRef Medline

7. Akoudad S, de Groot M, Koudstaal PJ, et al. Cerebral microbleeds are related to loss of white matter structural integrity. Neurology 2013;81:1930-37 CrossRef Medline

8. Reijmer YD, Freeze WM, Leemans A, et al; Utrecht Vascular Cognitive Impairment Study Group. The effect of lacunar infarcts on white matter tract integrity. Stroke 2013;44:2019-21 CrossRef Medline

9. Han F, Zhai FF, Wang Q, et al. Prevalence and risk factors of cerebral small vessel disease in a Chinese population-based sample. $J$ Stroke 2018;20:239-46 CrossRef Medline

10. Wardlaw JM, Smith EE, Biessels GJ, et al; STandards for ReportIng Vascular changes on nEuroimaging (STRIVE v1). Neuroimaging standards for research into small vessel disease and its contribution to ageing and neurodegeneration. Lancet Neurol 2013;12:82238 CrossRef Medline

11. Schmidt P, Gaser C, Arsic M, et al. An automated tool for detection of FLAIR-hyperintense white-matter lesions in multiple sclerosis. Neuroimage 2012;59:3774-83 CrossRef Medline

12. Cui Z, Zhong S, Xu P, et al. PANDA: a pipeline toolbox for analyzing brain diffusion images. Front Hum Neurosci 2013;7:42 CrossRef Medline

13. Smith SM, Jenkinson M, Johansen-Berg H, et al. Tract-based spatial statistics: voxelwise analysis of multi-subject diffusion data. Neuroimage 2006;31:1487-1505 CrossRef Medline
14. Mori S, Crain BJ, Chacko VP, et al. Three-dimensional tracking of axonal projections in the brain by magnetic resonance imaging. Ann Neurol 1999;45:265-69 CrossRef Medline

15. Colby JB, Soderberg L, Lebel C, et al. Along-tract statistics allow for enhanced tractography analysis. Neuroimage 2012;59:3227-42 CrossRef Medline

16. Song SK, Yoshino J, Le TQ, et al. Demyelination increases radial diffusivity in corpus callosum of mouse brain. Neuroimage 2005; 26:132-40 CrossRef Medline

17. van Veluw SJ, Reijmer YD, van der Kouwe AJ, et al. Histopathology of diffusion imaging abnormalities in cerebral amyloid angiopathy. Neurology 2019;92:e933-43 CrossRef Medline

18. D'Souza MM, Gorthi SP, Vadwala K, et al. Diffusion tensor tractography in cerebral small vessel disease: correlation with cognitive function. Neuroradiol J 2018;31:83-89 CrossRef Medline

19. Schreiber S, Bueche CZ, Garz C, et al. Blood brain barrier breakdown as the starting point of cerebral small vessel disease? New insights from a rat model. Exp Transl Stroke Med 2013;5:4 CrossRef Medline

20. Gons RA, de Laat KF, van Norden AG, et al. Hypertension and cerebral diffusion tensor imaging in small vessel disease. Stroke 2010; 41:2801-06 CrossRef Medline

21. Gons RA, van Norden AG, de Laat KF, et al. Cigarette smoking is associated with reduced microstructural integrity of cerebral white matter. Brain 2011;134:2116-24 CrossRef Medline

22. Heye AK, Thrippleton MJ, Chappell FM, et al. Blood pressure and sodium: association with MRI markers in cerebral small vessel disease. J Cereb Blood Flow Metab 2016;36:264-74 CrossRef Medline 